\title{
L'engagement militant dans la recherche en agriculture urbaine. Réflexions sur le contexte français au miroir du scholar activism nord-américain
} \author{
et Monique Poulot ${ }^{3}$ (D) \\ ${ }^{1}$ Géographie, Université de Lausanne, Institut de géographie et durabilité, Lausanne, Suisse \\ 2 Économie, Université de Lausanne, Institut de géographie et durabilité, Lausanne, Suisse \\ ${ }^{3}$ Géographie, Université Paris Nanterre, UMR LAVUE, Paris, France
}

Joëlle Salomon Cavin $^{1,{ }^{*}\left(\mathbb{D}, \text { Valérie Boisvert }^{2} \text { (D), Simone Ranocchiari }\right.}{ }^{1}$ (D), Quentin Dusserre-Bresson ${ }^{1}$

Reçu le 22 juillet 2019. Accepté le 5 novembre 2020

Cet article retrace la montée et l'évolution du militantisme académique parmi les géographes travaillant sur l'agriculture urbaine aux États-Unis et en France. Le militantisme académique se caractérise par l'engagement des chercheurs à soutenir les luttes des communautés socialement marginalisées à travers des recherches collaboratives, des publications et d'autres activités. Cette immersion au sein d'une communauté épistémique de géographes universitaires radicaux et féministes permet de souligner les différences majeures entre les conceptions françaises et américaines de l'engagement public, de l'implication communautaire et de la pratique scientifique, autant de défis spécifiques pour les chercheurs français voulant s'engager dans le militantisme académique. Cet article met en lumière le contraste de ces approches et les opportunités pour les chercheurs français de construire leurs propres modalités de militantisme académique dans la recherche en agriculture urbaine.

La Rédaction

Résumé - Cet article propose une réflexion collective sur l'engagement militant à partir de la recherche en agriculture urbaine en France et aux États-Unis. Il s'appuie sur une revue qualitative de la littérature, complétée par une série de séminaires. Ancrée dans la géographie critique et militante, la recherche sur l'agriculture urbaine aux États-Unis se concentre sur le jardinage communautaire et promeut la justice alimentaire. Dans le contexte français, les registres espaces et acteurs considérés sont beaucoup plus diversifiés et la réflexivité critique et politique comparativement moins développée. L'article se conclut par un appel à plus de réflexivité critique en ce domaine.

Mots clés : recherche / ville / agriculture urbaine / cultures épistémiques / engagement

\begin{abstract}
Activist engagement in urban agriculture research. Reflections on the French context in the mirror of North American scholar activism. This article is a collective reflection on how militant commitment shapes urban agriculture research in France and in the United States. It is based on a qualitative literature review complemented by a series of seminars and interviews devoted to researchers' experiences of engagement. In the United States, researchers who define themselves as activists clearly refer to critical and radical traditions of geography. Scholar activism is associated with identified epistemic communities and designates relatively codified ways of conceiving research to serve community struggles and projects. In France, the political commitment of intellectuals is above all an individual affair. Research on urban agriculture exemplifies these differences. In the United States it is largely conducted by scholaractivists and focuses on community gardening, and promotes the overall goal of food justice. In France, it borrows from various registers and focuses on much more diversified spaces and actors, and is globally marked by a lack of critical and political reflexivity. The article concludes with a call for more reflexivity in clarifying the ontological and epistemological assumptions of this field of research.
\end{abstract}

Keywords: research / city / urban agriculture / epistemic cultures / activism

\footnotetext{
* Auteur correspondant : joelle.salomoncavin@unil.ch
} 
Encadré 1. Sur la genèse de l'article et ses auteurs.

Cet article est le produit d'un projet de recherche intitulé «Quelles formes d'engagement des chercheurs dans l'agriculture urbaine?» financé par la Faculté des géosciences et de l'environnement de l'Université de Lausanne, coordonné par Joëlle Salomon Cavin avec Valérie Boisvert et Quentin Dusserre-Bresson (Institut de géographie et de durabilité, Université de Lausanne), Monique Poulot (UMR LAVUE, Université Paris-Nanterre) et Alexandre Aebi (Laboratoire de biodiversité des sols, Université de Neuchâtel).

Ce projet a été alimenté par plusieurs séminaires et rencontres qui ont été autant d'occasions d'échanger sur le thème de l'engagement:

- «Une recherche environnementale engagée », Université de Lausanne, 27 avril 2018, avec Hélène Bougouin, Leïla Chakroun, Nathalie Chèvre, Gabriel Cotte, Caroline Lejeune, Michel Jaboyedoff, Stuart Lane, Martine Rebetez, Benjamin Rudaz; https://wp.unil.ch/societenature/2018/04/une-recherche-environnementale-engagee/

- «Trait d'Urbain: une recherche en études urbaines engagée», Université de Lausanne, 25 mai 2018, avec Muriel Delabarre, Benoît Dugua, Florent Joerin et Martin Müller;

- «Quelles postures d'engagement des enseignants-chercheurs en agriculture urbaine?», Journée organisée à l'Université Paris-Nanterre, 20 Juin 2018, avec Alioune Badara Dabo, Ségolène Darly, Damien Deville, Giulia Giacchè, Monique Poulot, Camille Robert-Bœuf et Kristin Reynolds et complétée par un entretien avec Christine Aubry, AgroParisTech, Paris.

Ce sont ainsi de multiples témoignages, livrés par des chercheurs de disciplines diverses (sciences de la terre, humanités environnementales, géographie, urbanisme...), qui sont à l'origine de la réflexion présentée ici. Ils ne concernent pas tous l'agriculture urbaine. Certains traitent aussi de la durabilité, de la transition écologique et plus globalement de l'environnement.

Le texte proposé ici n'engage toutefois que ses auteurs, qui sont le «nous» qui s'y exprime. Joëlle Salomon Cavin mène des recherches sur l'agriculture urbaine et les relations ville-nature. Valérie Boisvert est économiste et travaille sur la conservation de la biodiversité. Simone Ranocchiari travaille sur des mouvements sociaux urbains en Italie après avoir pris part lui-même à leurs mobilisations. Quentin Dusserre-Bresson a réalisé une thèse sur les modes d'habiter périurbain et est membre fondateur d'une coopérative agricole à Genève. Monique Poulot est spécialiste des questions d'intégration de l'agriculture et de la nature dans le projet urbain.

L'idée de travailler sur l'engagement dans la recherche sur l'agriculture urbaine ${ }^{1}$ est d'abord née d'un choc culturel à l'une des conférences annuelles de l'Association américaine des géographes (AAG). Lors de l'édition 2018, un des organisateurs d'une session consacrée à l'agriculture urbaine, Hank Herrera, la soixantaine, de longs cheveux tressés, invite l'assemblée à se lever et à s'unir dans une prière en mémoire de la souffrance des esclaves et des Indiens d'Amérique. Se présentant comme un « chercheur activiste de couleur» qui travaille à favoriser l'accès de populations à faibles revenus à des aliments frais, sains, abordables et locaux (Bradley et Herrera, 2016), il dénonce à partir de son expérience le caractère prédateur de certaines recherches menées dans des jardins communautaires et livre un vibrant plaidoyer pour l'immersion des chercheurs dans les lieux et les collectifs qu'ils étudient (Herrera, 2018). La forme adoptée, aux antipodes des conventions comparativement policées qui régissent les échanges académiques sur le même sujet en France ou en Suisse, les mots employés - race, colonisation, patriarcat, suprématie...-, les références théoriques mobilisées - études postcoloniales, géographie féministe...-, l'ensemble participe d'un travail politique où la visée militante est revendiquée.

\footnotetext{
${ }^{1}$ Par agriculture urbaine, nous entendons de manière large toute pratique de production agricole et d'élevage dans et autour de la ville.
}

Cette session constitue la rencontre annuelle du groupe de travail «Food Justice Scholar-Activists/ Activist-Scholars Community of Practice» coordonné par Kristin Reynolds (Reynolds et al., 2018). Au-delà du moment évoqué plus haut, elle témoigne de la posture de toute une communauté de recherche.

Le projet à l'origine du présent article (Encadré 1) est le fruit d'expériences répétées de ce type, de confrontation à des répertoires d'expression, des pratiques de recherche et des modes d'engagement avec la société civile, inédits et déroutants dans le monde francophone européen. Il a également été suscité par des injonctions et des assignations de plus en plus fréquentes - émanant de médias, d'activistes, d'étudiantes et d'étudiants, voire de collègues - à produire des jugements, à délivrer des recommandations, à soutenir des projets ou des acteurs. Ces requêtes sont parfois formulées sur un mode particulièrement naiff, apolitique et acritique comme une demande de messages simples et forts, au nom, par exemple, de la durabilité et d'un impératif à agir face à l'urgence écologique. À l'inverse, elles peuvent prendre la forme d'un appel pressant à prendre parti, à fournir des ressources et des savoirs susceptibles de nourrir des luttes, à se faire entrepreneurs de causes. La question de l'engagement se pose ainsi à nous de façon récurrente si bien que nous avions envie de mener une réflexion commune sur ce thème, illustrée ici par la recherche en agriculture urbaine qui nous semble particulièrement 
emblématique de ce que nous cherchions à montrer, même si nous n'y sommes pas toutes et tous investis de la même façon.

Au-delà de ce thème et du champ de la géographie qui nous réunit, ce texte se nourrit de la diversité de nos regards et parcours. Il s'appuie sur une revue qualitative de la littérature relative à l'engagement en général, au militantisme académique ${ }^{2}$ nord-américain et à ses applications à la recherche sur l'agriculture urbaine en particulier. En outre, trois séminaires consacrés aux expériences d'engagement respectivement en sciences de l'environnement, dans les études urbaines, et en agriculture urbaine complétés par quelques entretiens nous ont permis de recueillir (en France et en Suisse romande) des récits d'engagement à la première personne, susceptibles d'éclairer la multiplicité des pratiques et des perceptions à cet égard.

Si l'idée générale de l'engagement est assez intuitive à saisir, les formes en sont multiples, depuis l'immersion dans des luttes politiques ou des mouvements sociaux afin de les comprendre de l'intérieur, à la production de plaidoyers pour leur compte, à un rôle de lanceur d'alerte ou de porte-parole de certaines causes, en passant par des recherches sur mandat et partenariales, etc... L'engagement est aussi une notion éminemment subjective pour les chercheurs qui l'affichent. Subjective au sens où elle est liée à un parcours de vie et de formation, à des valeurs personnelles; subjective aussi parce qu'elle relève du sentiment d'avoir dévié de certaines normes académiques pour défendre ses valeurs (écart plus ou moins perceptible depuis d'autres disciplines ou à l'aune d'autres critères). Le choix d'une sous-discipline ou d'un objet d'étude particulier - même abordé de façon relativement conventionnelle- peut ainsi être vécu comme un engagement par celui ou celle qui le fait. Il est donc difficile de donner une définition surplombante et parfaitement englobante de l'engagement.

Nous nous intéressons ici à la place de l'engagement militant dans la recherche en agriculture urbaine et cherchons à illustrer en quoi les communautés épistémiques et les épistémologies liées à l'agriculture urbaine diffèrent entre les États-Unis et la France. Il ne s'agit pas de traiter de façon exhaustive ni systématique des recherches menées dans ces deux pays, qui sont évidemment diverses, mais plutôt de rendre compte de

\footnotetext{
${ }^{2}$ Nous traduisons scholar activism, par militantisme académique qui en est la traduction la plus fréquente en français, pour traiter de l'engagement militant de chercheurs tel qu'il se manifeste dans leur activité de recherche, et éventuellement d'enseignement, au sein d'institutions universitaires mais aussi en dehors de celles-ci. Ce vocable est donc utilisé ici pour rendre compte d'un engagement directement lié au domaine de compétence des chercheurs, qui oriente et façonne leurs recherches.
}

grandes tendances et d'approches dominantes. Notre propos est d'esquisser des explications à ces différences et d'en explorer les implications en termes de pratiques, de productions et de mobilisations de la recherche. Après avoir dressé une généalogie du militantisme académique aux États-Unis, nous abordons le champ particulier des recherches en agriculture urbaine. Des exemples relatifs à la France viendront dans chaque partie apporter un contrepoint et un éclairage comparatif.

\section{Généalogie du militantisme académique (scholar activism)}

Les chercheurs qui se définissent comme militants (scholar activists) en agriculture urbaine aux États-Unis se réfèrent très clairement aux traditions critiques les plus ancrées dans ce pays: principalement la géographie radicale, notamment les travaux de David Harvey, les études féministes et les études postcoloniales (Fals-Borda et Rahman, 1991). Leur travail sur la justice environnementale et la justice alimentaire s'inscrit dans la continuité historique de la lutte pour les droits civiques (Torre et Fine, 2011) et la défense des minorités ethniques (Pulido, 2008). Une telle généalogie est impossible à tracer dans le contexte français où tant les pratiques actuelles que les répertoires plus traditionnels d'engagement des intellectuels sont différents (Denis, 2000).

Jusqu'à la fin des purges maccarthystes des années 1950, l'expression d'idées communistes, socialistes ou anarchistes, dont l'héritage est aujourd'hui revendiqué par certains chercheurs radicaux, était prohibée sur les campus américains (Ollman et Vernoff, 1984). La fin des années 1960 est marquée par de fortes mobilisations sociales et politiques et la «New Left» s'affirme en revendiquant droits civiques, libération des mœurs et une plus grande justice sociale, et en s'opposant à l'impérialisme et à la guerre du Vietnam (Granjon, 1988). Ces mouvements ont une influence majeure sur la recherche (DuBois et al., 1985, p. 1) avec la création d'associations radicales dans différentes disciplines par des chercheurs et surtout des étudiants qui aspirent à les réformer et à remettre en cause leurs relations avec le pouvoir et leur rôle dans les politiques impérialistes (Ollman et Vernoff, 1984). En 1969, Richard Peet fonde avec quelques collègues issus de ces mouvements la revue de « géographie radicale» Antipode (Jacoby, 1987, p. 181). Cette revue sert de catalyseur à l'émergence d'un courant de gauche au sein de la géographie, avant de prendre au début des années 1970 un tournant résolument marxiste à la suite des travaux de David Harvey. Les auteurs qui se réclament de la géographie radicale s'intéressent à la façon dont l'espace est produit par le capital et reflète des rapports d'exploitation. Selon Harvey (2012, p. 220), l'expression "géographie radicale»a été 
choisie car elle permettait d'englober la diversité des cultures politiques (marxistes, anarchistes etc.) de ses membres.

La réévaluation critique des catégories de pensée dominantes en sciences sociales constitue également la visée des études féministes, notamment la géographie féministe de J. K. Gibson-Graham, qui considère que l'objet de la recherche est de produire des changements individuels et sociétaux (Gibson-Graham, 2008). Les études féministes contribuent en outre à un renouvellement des modalités de production du savoir, en revendiquant la nécessité de repenser les frontières disciplinaires (Friedman, 2001). Combinées aux théories postcoloniales, elles ouvrent la voie à une approche plus globale des dynamiques qui produisent et entretiennent la sujétion et à un profond renouvellement épistémologique, à travers notamment le concept d'intersectionnalité (Crenshaw, 1991). Selon Michaël Löwy (2012, p. 12), il s'agit dorénavant de «penser ensemble, dans leur interdépendance, les subalternités multiples, les histoires de domination et les traditions de résistance souvent discordantes ».

À partir des années 1980, et tandis que la gauche non universitaire (partis, syndicats, mouvements sociaux, etc.) essuie des défaites, les courants dits radicaux s'institutionnalisent. Les publications se multiplient, de même que les cursus universitaires, et les chercheurs de gauche y trouvent de plus en plus d'opportunités de «professionnalisation» (Piven, 2010; Mitchell, 2004). Dans ce contexte, émerge la géographie dite «critique » (Gintrac, 2012), comparativement plus éclectique que la géographie radicale et intégrant une large coalition d'approches géographiques progressistes de gauche: géographie des minorités, féministe, postcoloniale, anarchisme, environnementalisme, études queer, situationnisme, poststructuralisme, etc. dont la revue ACME, «An International E-Journal for Critical Geographies», est emblématique. Le marxisme n'est plus le langage unique ni dominant de la contestation, au point, selon certains commentateurs, d'y perdre sa force politique et sa puissance agissante (Castree, 2000). L'intégration croissante des chercheurs radicaux aux institutions universitaires affecte également leurs registres d'expression puisqu'elle les incite à privilégier la critique académique comme forme suprême voire unique de critique politique et les déconnecte en partie des mouvements militants et des luttes sociales. La géographie critique devient ainsi un champ de spécialisation disciplinaire, régi par une codification qui lui est propre mais avec des règles et une grammaire plus académiques que militantes et dont le potentiel subversif est dès lors discuté (Castree, 2000). Le sociologue français Erik Neveu (2003, p. 118), évoque un « militantisme de campus » et la conviction affichée par certains chercheurs d'effectuer un travail politique majeur par la seule déconstruction des discours dominants.
Dans ce contexte, pour lutter contre la récupération, l'absorption de critiques militantes par le capitalisme et l'université néolibérale, un nombre croissant de chercheurs en appelle à la suite du géographe britannique Paul Routledge (1996, p. 402) à développer un tiers espace (third space), soit des espaces de résistance entre monde académique et activisme, dans lesquels les personnes appartenant à ces deux mondes apprendraient à intervertir leurs positions. Le militantisme académique consiste ainsi à estomper les frontières entre recherche et activisme dans la production des savoirs, à fournir de l'énergie réflexive aux mouvements sociaux, à orienter explicitement la recherche sur les objets de leurs luttes et à « commencer par ce que la communauté sait $»^{3}$ (Block et al., 2012, p. 213). Les ressources et la liberté offertes par un poste stable dans une université doivent être utilisées pour contester toutes les formes et structures dominantes - linguistiques, discursives, idéologiques et créer un contexte propice à la transformation sociale (Mitchell, 2004, p. 26). De sa conception à la diffusion de ses résultats, la recherche militante doit être menée en partenariat avec les personnes ou groupes soumis à cette souffrance afin de leur permettre de formuler et de mettre en œuvre des stratégies d'émancipation (Hale, 2001). Elle doit au final produire des savoirs d'action.

Aux États-Unis, loin d'être une appellation floue, le militantisme académique renvoie à des communautés épistémiques identifiées et désigne des façons relativement codifiées de concevoir et conduire la recherche pour servir des luttes et des projets communautaires (Hale, 2001). Des manuels, publications méthodologiques et forums d'échange lui sont consacrés (Hale, 2008; Bookchin et al., 2013; Conférence annuelle «Abriendo Brecha. Opening a path for activist scholarship » à l'Université du Texas à Austin); des groupes dédiés sont constitués dans les associations de chercheurs, à l'image de l'AAG $^{4}$.

En France, si la tradition d'engagement des intellectuels est très ancrée, il s'agit souvent jusqu'à une

\footnotetext{
${ }^{3}$ Le militantisme académique est très étroitement défini en relation avec la notion de "communauté» qui revêt dans ce contexte particulier le sens de groupe de personnes défavorisées, soumises à différentes formes de dominations et de discriminations, issues de minorités et en lutte pour la reconnaissance ou la conquête de droits, en l'occurrence à la ville et à l'alimentation. Autant de problèmes qui, dans le contexte français, seraient sans doute abordés plutôt dans leur dimension spatiale, avec comme unité d'analyse et de mobilisation les quartiers dits «sensibles».

${ }^{4} \mathrm{Si}$ notre propos est ici centré sur la géographie, le militantisme académique a une tradition bien ancrée également dans d'autres disciplines telles l'anthropologie ou l'archéologie (voir par exemple Tax, 1975 ; Bennett, 1996; Mullins, 2011 et la revue Collaborative Anthropologies, fondée en 2008).
} 
période récente d'un engagement politique partisan (Denis, 2000). Du fait de la prégnance des partis dans la vie politique et des conceptions et pratiques très différentes de la citoyenneté ordinaire, le travail politique des chercheurs ne peut prendre les mêmes formes qu'aux États-Unis. La culture universaliste française soustendue par l'intégration égalitaire et républicaine implique notamment l'emploi de catégories aveugles aux origines ethniques, à la couleur de peau, à la religion et à diverses formes d'identités minoritaires. De surcroît, le terme « communautés », dans les contours et l'usage de ce terme aux États-Unis, c'est-à-dire qui porteraient seules la responsabilité politique de leur devenir, n'est guère envisageable. La tradition de l'État-providence, même chancelant, et de politiques publiques structurant l'espace et ses usages reste ancrée, de sorte que les revendications et les grammaires critiques sont d'un autre ordre. Ainsi la question de l'intervention collective des chercheurs dans l'espace public telle qu'elle se pose aux États-Unis est relativement récente, les réponses apportées sont plus individuelles et diversifiées, moins théorisées et codifiées.

L'essor de la pensée contestataire à l'origine des courants radicaux actuels au sein des sciences humaines et sociales est largement lié à Mai 68 et aux débats politiques et idéologiques afférents, avec un arrière-plan marxiste surdéterminant. D'une part, de nouvelles postures d'intervention s'affirment quand des intellectuels s'érigent en défenseurs de certains groupes ou causes et se font les «compagnons de route» de partis politiques (Denis, 2000, p. 10); de l'autre de nouvelles universités émergent, qui adoptent des projets pédagogiques différents, comme le centre universitaire expérimental de Vincennes ${ }^{5}$. En matière d'épistémologie, des ruptures s'opèrent avec le développement des approches postmodernes et les travaux de Foucault, Deleuze, Derrida, Lyotard, Baudrillard ou Guattari, en sciences humaines, en lettres et en philosophie, mais la géographie peu affectée alors par ce renouveau n'y joue pas un rôle de premier plan. Dans les années 1970, l'engagement politique est en outre surtout une affaire individuelle: il émane moins de chercheurs affirmant leur origine disciplinaire et une forme d'ethos lié à celleci que d' «intellectuels», qui mettent leur légitimité académique ou leur aura médiatique personnelles au

\footnotetext{
${ }^{5}$ Créé en novembre 1968, le centre universitaire expérimental de Vincennes s'est notamment constitué autour du projet de politiser les pratiques pédagogiques, en abolissant les cours magistraux et les examens terminaux, en s'ouvrant aux nonbacheliers et aux salariés, en valorisant la prise de parole et la remise en cause de l'autorité de l'enseignant, en recrutant par cooptation des enseignants choisis pour leur proximité avec l'avant-garde intellectuelle et leurs engagements politiques à gauche (Soulié, 2012).
}

service de combats divers (Denis, 2000, p. 20-21). Foucault (2010c) distingue ainsi «intellectuels universels» et «intellectuels spécifiques». Les premiers diraient «le juste-et-le-vrai pour tous», cherchant à s'ériger en conscience universelle, tandis que les seconds se politisent dans les secteurs où ils disposent de savoirs, où les situent leurs conditions de travail ou de vie, ce qui selon Foucault leur permettrait de gagner «une conscience beaucoup plus concrète et immédiate des luttes » (Foucault, 2010a; 2010b; 2010c). Dans un cas comme dans l'autre, il s'agit moins de mettre au service de causes un outillage conceptuel appliqué avec rigueur, que de se prévaloir de celui-ci pour asseoir une légitimité personnelle à intervenir dans le débat public. Si les figures d'intellectuels engagés abondent dans l'espace médiatique français, elles sont assez éloignées du travail militant livré par les géographes américains avec des minorités et populations en lutte pour leurs droits.

Pour trouver en France des échos au militantisme académique d'outre-Atlantique, il faut s'intéresser à des débats bien plus récents sur l'engagement, l'implication voire l'embarquement qui posent les questions fondamentales de la subjectivité, de la neutralité axiologique et de l'autonomie des chercheurs vis-à-vis des objets et des mouvements sociaux étudiés. La globalisation, la diffusion généralisée de la doxa néolibérale, le déclin des partis dans la vie politique, et parallèlement le développement de nouveaux mouvements sociaux et registres de mobilisation ouvrent de nouveaux espaces de réflexion sur ces thèmes en sciences sociales.

Née en France dans les années 1960, la géographie sociale a ainsi été pensée dès son origine comme une approche critique et impliquée (Séchet et Veschambre, 2006). Elle a été influencée par les épistémologies critiques venues d'Outre-Atlantique évoquées plus haut dans les années 1990-2000 (Collignon, 2001), dans le sillage du postmodernisme, en lien avec des questions urbaines, sociales et environnementales. La notion de justice spatiale est désormais fortement mobilisée dans les études urbaines, devenues le lieu privilégié d'analyse des travers du néolibéralisme, et dont le «Droit à la ville» de Lefebvre (1968) constitue une référence incontournable (Gintrac et Giroud, 2014). En témoigne la revue bilingue et interdisciplinaire Justice spatiale/ spatial justice, créée en 2009 à l'Université de Nanterre, qui se réfère explicitement à David Harvey et Edward Soja ${ }^{6}$.

D'autres champs se développent en France dans le prolongement ou en réaction aux courants critiques anglophones comme la géographie postcoloniale (Collignon, 2007), celle du genre (Hancock, 2002) ou encore la

\footnotetext{
${ }^{6}$ Voir le dossier «Ville, néolibéralisation et justice» en juin 2014 : www.jssj.org/issue/mai-2014-dossier-thematique/.
} 
géographie queer (Borghi, 2014), mais aussi la géographie environnementale et la political ecology (Chartier et Rodary, 2016). Tous participent d'une dénonciation des rapports de domination dans la société en général et dans la géographie en particulier, mais traitant d'une multitude d'objets, à l'aide de méthodes et cadrages multiples, ils ne constituent pas un courant unifié aisément repérable (Morange et Calberac, 2012). Accordant une primauté au travail empirique, ces approches partagent une certaine défiance à l'égard des totalisations théoriques et des analyses binaires des formes de domination (Morange et Calberac, 2012) avec une prise de distance vis-à-vis de la géographie marxiste des années 1960-1980, et par ricochet des géographies critiques institutionnalisées des États-Unis dans lesquelles la théorie joue un rôle structurant.

L'intérêt pour l'environnement amène quant à lui des formes spécifiques de décentrement du regard. Il favorise le développement de nouvelles communautés épistémiques, de champs transdisciplinaires, d'approches impliquées et sensibles de collectifs et d'objets auxquels est reconnue une certaine agentivité. Les rapports entre sociétés et nature sont réévalués, de nouvelles ontologies de la nature qui rompent avec les catégories de la pensée moderne sont envisagées. Cela favorise une redéfinition des praxis de recherche et des normes de qualité mettant l'accent sur la réflexivité.

Aussi bien les motivations que les figures de l'engagement sont ainsi multiples dans les géographies critiques «à la française » (Morange et Calberac, 2012). $\mathrm{Si}$ l'objectif de dévoilement des violences et des exclusions de tout ordre est globalement partagé, l'engagement des chercheurs apparaît plutôt comme une affaire de sensibilité individuelle, souvent liée au terrain et commandée par l'objet de recherche (Bautès et Marie dit Chirot, 2012) ou les combats et valeurs personnels des chercheurs. L'agriculture urbaine en tant que champ de recherche est emblématique de ces évolutions récentes dans la mesure où elle participe à une redéfinition des rapports à la nature, à la terre et à la production et où elle est associée à des projets et des revendications sociales.

\section{La recherche militante en agriculture urbaine}

Les études consacrées à l'agriculture urbaine aux États-Unis et en France reflètent les différences de culture militante dans la recherche, auxquelles s'ajoute la complexité relative à cet objet particulier. Ainsi ces études renvoient-elles aux différences majeures de réalités urbaines, de rapports à l'agriculture et à l'alimentation, de conceptions et de pratiques de l'espace public et de la citoyenneté, de même qu'aux disciplines d'origine des chercheurs. Tandis qu'aux États-Unis les recherches en agriculture urbaine sont dominées par le militantisme académique - bien qu'évidemment pas limitées à celui-ci - et se concentrent sur le jardinage communautaire, elles empruntent à des registres et se consacrent à des pratiques, des espaces et des acteurs beaucoup plus diversifiés en France ${ }^{7}$.

La recherche militante en agriculture urbaine aux États-Unis s'inscrit dans la visée générale de la justice alimentaire (Gottlieb et Joshi, 2010). Elle considère que l'agriculture urbaine, suivant la façon dont elle est pratiquée et étudiée, peut aussi bien être radicale ou réformiste que relever d'un projet néolibéral (Tornaghi, 2014). S'y investir peut représenter un acte de résistance ou un prolongement de formes structurelles d'oppression. Les chercheurs se veulent à la fois critiques et constructifs (McClintock, 2014) et poursuivent un double agenda: d'une part, dénoncer ce qui participe à la reproduction des inégalités sociales, d'autre part, contribuer à faire de l'agriculture urbaine un levier d'émancipation pour des populations défavorisées.

Dans une perspective critique, ils s'attachent à démontrer que les initiatives de jardinage urbain promues dans les médias et soutenues par les pouvoirs publics sont plutôt portées par des populations blanches fortement dotées en capital social. L'ouvrage Beyond the kale de Kristin Reynolds et Nevin Cohen (2016) appelle précisément, comme son titre l'indique, à aller au-delà de ces pratiques supposément nouvelles. A contrario, les auteurs entendent souligner qu'à New York, le jardinage urbain n'a jamais cessé d'être pratiqué par des populations de couleur à faibles revenus mais que ce jardinage a été rendu invisible par un cadrage et des modes d'évaluation qui tendent à disqualifier ces projets, jugés à l'aune de conceptions du succès qui ne sont pas les leurs. L'agriculture urbaine constitue donc aussi un terrain de reproduction de l'altérité raciale et de réaffirmation, voire d'extension de logiques de domination de classes et de genre qu'il convient de mettre explicitement au jour (Reynolds et Cohen, 2016).

Dans un souci d'équilibre et de justice sociale, les chercheurs militants en agriculture urbaine s'assignent un objectif de discrimination positive dans le choix des projets qu'ils soutiennent en même temps qu'ils les étudient, donnant une visibilité aux expériences impliquant des

\footnotetext{
${ }^{7}$ Nous sommes pleinement conscientes et conscients que toute la recherche en géographie sur l'agriculture urbaine dans les deux pays ne relève pas des étiquettes que nous lui avons accolées et qu'on peut repérer des travaux traitant de durabilité ou de services écosystémiques rendus par l'agriculture en ville sous un angle très technique et distancié des réalités sociales et politiques aux États-Unis, aussi bien que des travaux engagés en France. Nous entendons mettre en avant des grandes tendances, et des poids et des niveaux contrastés d'institutionnalisation du militantisme académique.
} 
personnes défavorisées afin de travailler sur et à un développement du pouvoir émancipateur de l'agriculture urbaine (Reynolds et Cohen, 2016). Le choix du terrain est posé comme acte politique et la démarche de recherche mise en œuvre répond aux principes du militantisme académique décrits plus haut. L'objectif est de produire des résultats qui puissent être mobilisés par les populations, en rupture avec les démarches de recherche sans retombées locales, qualifiées d'extractives (Bradley et Herrera, 2016; Slocum et al., 2016). Cela suppose un intérêt particulier porté aux besoins de la population, une proximité et une immersion intime et concrète dans les mouvements auxquels ils entendent contribuer (Piven, 2010). La démarche de recherche est expressément fondée sur la réciprocité et les échanges, tant pratiques qu'intellectuels, entre les chercheurs et les membres de la «communauté ». Ces derniers, loin d'être réduits à un rôle passif de sources d'information ou de victimes démunies d'une histoire écrasante, pour lesquelles il s'agirait d'obtenir réparation (Tuck, 2009), sont reconnus comme détenteurs actifs de savoirs, de talents et de capacités d'agir.

Les méthodes privilégiées relèvent donc de la recherche-action participative critique (Block et al., 2012). Une attention particulière est portée aux mécanismes par lesquels les rapports de domination et autres formes d'injustice spatiale et alimentaire sont à la fois occultés et entretenus. Surtout, les procédures et les critères d'évaluation des projets doivent être redéfinis pour porter un regard plus juste sur les différents types d'expériences, sans réduire leur altérité à différents niveaux de performance. Cela conduit les chercheurs à s'engager dans le débat politique, à porter et à défendre leurs résultats, à se mobiliser activement aux côtés des populations défavorisées: ce que Laura Pulido qualifie de «talk plus walk» pour souligner la différence avec un militantisme de campus qui miserait uniquement sur la puissance performative du discours académique comme force de changement.

Ces pratiques particulières de recherche sont toutefois souvent liées à des rattachements institutionnels qui accordent aux chercheurs une grande liberté. La recherche militante en agriculture urbaine est largement menée au sein d'universités publiques (The State University of New York, The City University of New York) qui ont des missions explicites de service aux communautés, au sens de service à la cité, et où s'est en grande partie construit et institutionnalisé le militantisme académique (par exemple à travers les travaux de Frances Fox Piven). De surcroît, dans un contexte de recul de l'État-providence, des fondations privées soutiennent les projets appliqués au service de la population et sont à l'origine d'une production importante de recherches contractuelles. La communauté épistémique de l'agriculture urbaine s'y investit massivement, ce qui explique le style des communications et des publications, marqué par une hybridation entre forme académique assez classique et registre politique communautaire. Les trajectoires au sein de cette communauté sont elles-mêmes souvent hybrides, entre activisme et monde académique, à l'exemple de Kristin Reynolds qui occupe un poste d'enseignement à l'Université, lui permettant d'assumer le rôle de formation et de transmission qu'elle juge primordial tout en réalisant sa recherche dans un cadre privé, distinct, lui laissant toute liberté de choisir ses projets. Un certain nombre de chercheurs en agriculture urbaine aux États-Unis sont en outre des activistes issus des minorités aux côtés desquelles ils s'engagent, tels Hank Herrera et Leah Penniman (2018). Pour certains d'entre eux, la recherche est une seconde carrière ou une carrière parallèle, entreprise après ou en marge d'activités plus directement liées aux services communautaires, dans les domaines du soin ou du travail social.

Les registres de partage d'expériences de recherche sur l'agriculture urbaine témoignent clairement de la porosité et de l'interpénétration des mondes académique et militant. Les réseaux se constituent autour de communautés de pratiques ou par affinités théoriques ou méthodologiques plutôt que selon les disciplines et réunissent des chercheurs-activistes et des activisteschercheurs (Reynolds et al., 2018) qui ont développé des espaces et un style d'expression qui leur sont propres. La présentation de soi, le récit à la première personne de parcours de recherche militants, l'exercice d'une réflexivité critique sous la forme de lettres ouvertes, de questions/réponses, inspirés des méthodes de pédagogie active et des échanges sur les réseaux sociaux y occupent une place importante.

Comme le militantisme académique de façon plus générale, cette approche de la recherche en agriculture urbaine diffuse progressivement en Europe continentale, comme l'illustre une série de publications récentes, mais sous des formes diverses, bien moins unifiées et sans toujours d'implication militante des chercheurs (Tornaghi et Certomà, 2019; Darly et McClintock, 2017 ; Hochedez et Le Gall, 2016; Rosol, 2012; Cahn et al., 2018). L'agriculture urbaine y est abordée sous un angle critique, dans des dimensions quotidiennes et micropolitiques, et resituée dans les perspectives plus larges de la justice sociale et alimentaire et de la ville néolibérale. Si les géographies critiques sont une source d'inspiration majeure pour aborder l'agriculture urbaine comme champ politique (Tornaghi et Certomà, 2019), à la diversité des objets répond une diversité d'approches puisant à plusieurs disciplines et méthodes.

En France, la plupart des recherches, majoritairement menées par des agronomes et des géographes, sont centrées sur l'agriculture urbaine en elle-même - et non comme entrée pour dénoncer l'exclusion, soutenir des luttes sociales ou produire des savoirs émancipateurs. 
Elles ne sont pas surdéterminées par un agenda militant ni par conséquent aussi sélectives dans le choix de leurs objets. Les expériences étudiées sont donc variées, du jardinage urbain à l'agriculture professionnelle périurbaine (Nahmias et Le Caro, 2012), en passant par les projets high-tech de fermes verticales et les initiatives de végétalisation citoyenne de l'espace public (Astigarraga, 2014). Les questions soulevées relèvent des fonctions et des formes de l'agriculture urbaine (Pourias et al., 2016), des rapports ville-campagne (Le Caro et al., 2016), de l'alimentation des villes (Perrin et Soulard, 2017; Lardon et Loudiyi, 2014) ou encore de l'agriculture dans le projet urbain (Vidal et Fleury, 2009; Poulot, 2014). À la différence des États-Unis, tous les aspects abordés ne sont pas régis sans partage ni nuance par une gouvernementalité néolibérale. À l'image de la diversité des thèmes traités, les références théoriques le sont tout autant: certains mobilisent le cadre conceptuel de la justice alimentaire (Hochedez et Le Gall, 2016 ; Paddeu, 2018), ou la notion de justice spatiale pour l'analyse des politiques foncières (Perrin et Nougarèdes, 2017). Les résultats et les formats de communication sont de facture comparativement plus classique qu'aux États-Unis et sont marqués, au-delà de la variété des approches, par un style plus académique. À la différence des États-Unis, les recherches sont le plus souvent financées par des fonds publics.

En France, certains des chercheurs qui travaillent sur l'agriculture urbaine ont une formation initiale en agronomie, et donc en ingénierie, d'où probablement des aspirations plus marquées à la neutralité axiologique que dans le milieu universitaire de sciences sociales (Soulard et al., 2018). Leurs recherches sont dès lors aussi guidées par un objectif d'amélioration, fondé sur des critères d'efficacité technique et de performance agronomique, à distance de contingences ouvertement politiques. De fait, la «culture d'ingénieur », telle qu'elle est développée en France, avec un système sui generis élitiste de formation au sein d'écoles, ne prépare guère à appréhender les acteurs des projets étudiés, qu'il s'agisse d'agriculteurs ou à plus forte raison d'activistes, comme des partenaires de la recherche, producteurs de savoirs. Elle incite à adopter une position d'expertise surplombante vis-à-vis de ceux qui sont perçus comme destinataires de la recherche (Soulard et al., 2018). C'est d'ailleurs une des raisons mentionnées par les chercheurs qui ont ressenti le besoin de se former ensuite en sciences sociales pour expliquer leur tournant disciplinaire, et finalement leur changement de métier, de l'ingénierie à la recherche.

Mais parallèlement, le fait de mener des recherches au sein d'écoles d'ingénieurs comme AgroParisTech autorise des modalités plutôt atypiques pour la France, à l'image du bureau d'expertise en agricultures urbaines créé par Christine Aubry. Cette structure, qui permet d'accueillir des fonds privés, développe des partenariats ad hoc avec des collectivités ou des entreprises privées autour de projets variés qui en appellent à l'expertise pluridisciplinaire des enseignants-chercheurs (en agronomie, écologie, bioclimatologie, géographie, sociologie et économie) et contribuent à la formation ou au perfectionnement par la pratique des élèves ingénieurs au conseil en agriculture urbaine. S'il s'agit bel et bien d'une recherche-action, elle est avant tout à finalité opérationnelle, inscrite dans des relations contractuelles et répondant à des commandes explicites. Elle s'apparente à une offre de prestations d'appui mais sans orientation militante a priori.

Compte tenu de l'importance et de l'expérience de l'équipe porteuse de ce projet, elle a contribué à l'adoption d'une acception très englobante de la recherche impliquée en agriculture urbaine et à la coexistence de pratiques, rationalités et épistémologies multiples, comme en témoigne la diversité des termes couramment employés : recherche-action participative, science participative, recherche impliquée engagée, etc. De même, les chercheurs en agriculture urbaine se conçoivent rarement explicitement comme militants ou activistes mais plutôt comme médiateurs, facilitateurs ou consultants (Soulard et al., 2018).

L'engagement défini dans ce sens inclusif fait aujourd'hui une percée notable dans la programmation scientifique des institutions de recherche, comme thème et pratique de recherche, en miroir au développement de nouvelles formes d'activisme et de mobilisations citoyennes, et en lien avec les réseaux internationaux de recherche sur l'agriculture urbaine. Compte tenu de la diversité des approches mentionnées, le terrain d'entente partagé est minimal : l'implication est présentée comme une nécessité pour saisir ce qui se joue à travers les pratiques d'agriculture urbaine et produire des savoirs susceptibles de conduire au changement, plus souvent défini en relation avec la transition écologique ou la ville durable qu'en termes d'émancipation. L'engagement à la française dans ses contours actuels serait ainsi à situer plus du côté d'un impératif de méthode que du côté de l'activisme.

$\mathrm{Au}$ final, alors que le sujet connaît un fort développement et soulève un grand engouement, il ne s'est pas constitué en France de communauté épistémique sur l'agriculture urbaine comparable à celle qui existe aux États-Unis et il n'y a pas eu de travail collectif d'élaboration d'un ethos de l'engagement.

\section{Conclusion}

L'agriculture urbaine apparaît comme un champ de recherche militant, particulièrement aux États-Unis où les chercheurs qui contribuent à son étude se définissent 
comme scholar activists et entendent contribuer aux luttes politiques pour la justice sociale et alimentaire et le droit à la ville de communautés avec lesquelles ils travaillent. Les dimensions normatives et politiques des recherches sur l'agriculture urbaine sont comparativement peu débattues en France et rarement mises en avant par les chercheurs. S'il existe une part de prosélytisme dans certains travaux qui s'attachent à promouvoir l'agriculture urbaine, il relève souvent plutôt d'un projet d'identification puis de diffusion de bonnes pratiques, sans analyse approfondie des espaces dans lesquels elles se déploient.

Certes, les écarts entre les pratiques de recherche en agriculture urbaine en France et aux États-Unis tendent à s'estomper en raison de la mobilité des chercheurs et de l'internationalisation de la recherche. Néanmoins, des cultures épistémiques nationales différentes liées aux trajectoires historiques singulières des pays persistent, contribuant à institutionnaliser différemment les approches militantes de recherche dans le champ académique. Le militantisme académique ne jouit pas de la même reconnaissance ni de la même légitimité en France et aux États-Unis. L'affirmation d'une subjectivité militante y est sans doute comparativement plus risquée pour un chercheur car moins lisible en termes de carrière. Dans des disciplines dominées par un idéal plus ou moins conscient d'objectivisme et de neutralité axiologique, la production de discours à la première personne peut largement dérouter. Les pratiques de recherche sont toutefois en train d'évoluer à la faveur du développement de champs qui, à des titres divers, transcendent les clivages entre mouvements académiques, militants, artistiques et contre-culturels. L'entrelacs des dimensions abordées requiert une recherche vécue et décrite comme performance, qui engage les valeurs, mais aussi les sens, les émotions et la corporalité, dans laquelle les distances entre sujets et objets s'abolissent, où les pratiques de recherche se fondent dans les pratiques étudiées. L'affirmation de la position adoptée par les chercheurs revêt dans ces contextes une importance majeure.

Concernant l'agriculture urbaine, et bien d'autres domaines à l'évidence, l'exercice de la réflexivité et la production de recherches ouvertement situées, qui rendent explicites leurs présupposés ontologiques et épistémiques, nous paraissent essentiels. Sortir de l'ambiguiité évoquée en introduction est indispensable: face à des sujets qui engagent et à des injonctions à prendre parti, il faudrait pouvoir rendre compte avec rigueur de ses propres modalités d'engagement, sans avoir à produire sur le moment une explication ad hoc. Au regard des différences de contexte mises en évidence, l'importation pure et simple des registres et des méthodes de l'activisme académique des États-Unis semble malaisée et en partie hors de propos. La forme de militantisme académique adaptée aux répertoires d'expression et d'action des collectifs avec lesquels nous travaillons et aux formes particulières de structuration des champs politique et académique qui sont les nôtres reste largement à construire et à codifier, chantier qui nous paraît essentiel et urgent.

\section{Références}

Astigarraga R., 2014. Écosphère : la végétalisation d'un espace public urbain pour l'émergence d'une vie de quartier ou comment habiter la ville autrement, Pour, 4, 224, 205-213. https://doi.org/10.3917/pour.224.0205.

Bautès N., Marie dit Chirot C., 2012. Pour une géographie sociale de l'action, Carnets de Géographes, 4, https://doi. org/10.4000/cdg.982.

Bennett J.W., 1996. Applied and action anthropology: ideological and conceptual aspects, Current Anthropology, 37, 1, Supplement, Special Issue: Anthropology in Public, 23-53, www.journals.uchicago.edu/doi/pdf/10.1086/204461.

Block D.R., Chávez N., Allen E., Ramirez D., 2012. Food sovereignty, urban food access, and food activism: contemplating the connections through examples from Chicago, Agriculture and Human Values, 29, 203-15, https://doi.org/10.1007/s10460-011-9336-8.

Bookchin N., Brown P., Ebrahimian S., Colectivo Enmedio, Juhasz A., Martin L., MTL, Mirzoeff N., Ross A., Saab A. J., Sitrin M. 2013. Militant Research Handbook, New York, New York University, www.visualculturenow.org/wpcontent/uploads/2013/09/MRH_Web.pdf.

Borghi R., 2014. L'espace à l'épōque du queer : contaminations queer dans la géographie française, PolitiQueer, numéro Dimensions francofolles, 14-26.

Bradley K., Herrera H., 2016. Decolonizing food justice: naming, resisting, and researching colonizing forces in the movement, Antipode, 48, 1, 97-114, https://doi.org/ 10.1111/anti.12165.

Cahn L., Deligne C., Pons-Rotbardt N., Prignot N., Zimmer A., Zitouni B., 2018. Terres des villes. Enquêtes potagères de Bruxelles aux premières saisons du $21^{e}$ siècle, Paris, Éditions de l'Éclat.

Castree N., 2000. Professionalisation, activism, and the university: whither 'critical geography'?, Environment and Planning A: Economy and Space, 32, 6, 955-970, https://doi.org/10.1068/a3263.

Chartier D., Rodary E. (Eds), 2016. Manifeste pour une géographie environnementale : géographie, écologie, politique, Paris, Presses de Sciences Po.

Collignon B., 2001. La géographie radicale à la recherche d'un nouveau souffle. Introduction, in Staszak J.-F. (Ed.), Géographies anglo-saxonnes. Tendances contemporaines, Paris, Belin, 131-138.

Collignon B., 2007. Note sur les fondements des postcolonial studies, EchoGéo, 1, https://doi.org/10.4000/echogeo.2089.

Crenshaw K., 1991. Mapping the margins: intersectionality, identity politics, and violence against women of color, Stanford Law Review, 43, 6, 1241-1299, http://www.jstor. org/stable/1229039. 
Darly S., McClintock N., 2017. Introduction to urban agriculture in the neoliberal city: critical European perspectives, ACME: An International Journal for Critical Geographies, 16, 2, 224-231, https://acme-journal.org/ index.php/acme/article/view/1598.

Denis B., 2000. Littérature et engagement : de Pascal à Sartre, Paris, Seuil.

DuBois E.C., Kelly G.P., Kennedy E.L., Korsmeyer C.W., Robinson L.S., 1985. Feminist scholarship: kindling in the groves of academe, Urbana, University of Illinois Press.

Fals-Borda O., Rahman M.A., 1991. Action and knowledge: breaking the monopoly with participatory action research, New-York, Apex Press.

Foucault M., 2010a. Je perçois l'intolérable, in Foucault M., Dits et écrits I, année 1971, 94, Paris, Gallimard, 1071-1073.

Foucault M., 2010b. Les intellectuels et le pouvoir, in Foucault M., Dits et écrits I, année 1972, 106, Paris, Gallimard, 1174-1183.

Foucault M., 2010c. La fonction politique de l'intellectuel, in Foucault M., Dits et écrits II, année 1976, 184, Paris, Gallimard, 109-114.

Friedman S.S., 2001. Statement. Academic feminism and interdisciplinarity, Feminist Studies, 27, 2, 504-509, https:// doi.org/10.2307/3178774.

Gibson-Graham J.K., 2008. Diverse economies: performative practices for 'other worlds', Progress in Human Geography, 32, 5, 613-632, https://doi.org/10.1177/0309132508090821.

Gintrac C., 2012. Géographie critique, géographie radicale: comment nommer la géographie engagée ?, Carnets de Géographes, 4, www.carnetsdegeographes.org/carnets_re cherches/rech_04_04_Gintrac.php.

Gintrac C., Giroud M. géographie critique de l'urbain, Paris, Les Prairies ordinaires.

Gottlieb R., Joshi A., 2010. Food justice, Cambridge (Mass)/ London, MIT Press.

Granjon M.-C., 1988. Révolte des campus et nouvelle gauche américaine (1960-1988), Matériaux pour l'histoire de notre temps, 11-13, 10-17, https://doi.org/10.3406/ mat.1988.403819.

Hale C.R., 2001. What is activist research?, Items \& Issues, 2, 1-2, 13-15, Social Sciences Research Council.

Hale C.R., 2008. Engaging contradictions. Theory, politics and methods of activist scholarship, Berkeley, University of California Press.

Hancock C., 2002. Genre et géographie : les apports des géographies de langue anglaise, Espace Populations Sociétés, 3, 257-264, https://doi.org/10.3406/espos.2002.2038.

Harvey D., 2012, Marx and the city, entretien avec David Harvey réalisé par Philippe Mangeot, Dominique Dupart, Cécile Gintrac et Nicolas Vieillescazes, Vacarme, 59, 2, 218-249, https://doi.org/10.3917/vaca.059.0218.

Herrera H., 2018. The value and meaning of experience in food system learning spaces: reflections from the activist and traditional community perspectives, ACME: An International Journal for Critical Geographies, 17, 4, 1085-1094, www.acme-journal.org/index.php/acme/article/view/1513.

Hochedez C., Le Gall J., 2016. Justice alimentaire et agriculture, Justice spatiale/Spatial Justice, 9, 1-31, http://www.jssj.org/ article/justice-alimentaire-et-agriculture/.
Jacoby R., 1987. The last intellectuals: American culture in the age of academe, New York, Basic Books.

Le Caro Y., Jousseaume V., Poulot M., Rouget N., 2016. Agricultures et villes : des articulations renouvelées, Annales de géographie, 712, 6, 553-563, https://doi.org/10.3917/ ag.712.0553.

Lardon S., Loudiyi S., 2014. Agriculture et alimentation urbaines: entre politiques publiques et initiatives locales, Géocarrefour, 89, 1-2, 3-10, http://journals.openedition. org/geocarrefour/9362.

Lefebvre H., 1968. Le droit à la ville, Paris, Anthropos.

Löwy M., 2012 [1 ${ }^{\text {re }}$ éd. 1985]. Les aventures de Karl Marx contre le baron de Münchhausen. Introduction à une sociologie critique de la connaissance, Paris, Syllepse.

McClintock N., 2014. Radical, reformist, and garden-variety neoliberal: coming to terms with urban agriculture's contradictions, Local Environment, 19, 2, 147-171, https://doi.org/10.1080/13549839.2012.752797.

Mitchell D., 2004. Radical scholarship: a polemic on making a difference outside the Academy, in Fuller D., Kitchin R. (Eds), Radical theory/critical praxis: making a difference beyond the Academy?, Victoria (B.C.), Praxis (e)Press, 2131, https://kitchin.org/wp-content/uploads/2020/05/ RTCP_Whole.pdf.

Morange M., Calberac Y., 2012. Géographies critiques «à la française »?, Carnets de géographes, 4, http://journals. openedition.org/cdg/976.

Mullins P.R., 2011. Practicing anthropology and the politics of engagement: 2010 year in review, American Anthropologist, $113,2,235-245$, https://doi.org/10.1111/ j.1548-1433.2011.01327.X.

Nahmias P., Le Caro Y., 2012. Pour une définition de l'agriculture urbaine: réciprocité fonctionnelle et diversité des formes spatiales, Environnement urbain/Urban Environment, 6, http://journals.openedition.org/eue/437.

Neveu É., 2003. Recherche et engagement: actualité d'une discussion, Questions de communication, 3, 109-120, https://doi.org/10.4000/questionsdecommunication.7469.

Ollman B., Vernoff E. (Eds), 1984. The Left academy: Marxist scholarship on American campuses, New York, Praeger.

Paddeu F., 2018. De l'agriculture urbaine à la justice alimentaire : regards critiques franco-américains. Entretien avec Nathan McClintock et Christophe-Toussaint Soulard, Urbanités, 10, www.revue-urbanites.fr/10-mcclintock-soulard/.

Penniman L., 2018. Farming while black. Soul fire farm's practical guide to liberation on the land, White River Junction, Chelsea Green.

Perrin C., Soulard C.-T., 2017. L'agriculture dans le système alimentaire urbain : continuités et innovations, Natures Sciences Sociétés, 25, 1, 3-6, https://doi.org/10.1051/nss/2017012.

Perrin C., Nougarèdes B., 2017. An analytical framework to consider social justice issues in farmland preservation on the urban fringe. Communication à la conférence internationale Global Rural Development and Land Capacity Building, 26 au 29 août, Yulin (Chine).

Piven F.F., 2010. Reflections on scholarship and activism, Antipode, 42, 4, 806-810, https://doi.org/10.1111/ j.1467-8330.2010.00776.x. 
Pulido L., 2008. FAQs: Frequently asked questions on being a scholar/activist, in Hale C.R. (Ed.), Engaging contradictions. Theory, politics and methods of activist scholarship, Berkeley, University of California Press, 341-366.

Poulot M., 2014. L'invention de l'agri-urbain en Île-de-France. Quand la ville se repense aussi autour de l'agriculture, Géocarrefour, 89, 1-2, 11-19, https://doi.org/10.4000/geocar refour.9363.

Pourias J., Aubry C., Duchemin E., 2016. Is food a motivation for urban gardeners? Multifunctionality and the relative importance of the food function in urban collective gardens of Paris and Montreal, Agriculture and Human Values, 33, 257-273, https://doi.org/10.1007/s10460-015-9606-y.

Reynolds K., Block D., Bradley K., 2018. Food justice scholaractivism and activist-scholarship. Working beyond dichotomies to deepen social justice praxis, ACME: An International Journal for Critical Geographies, 17, 4, 988-998, www. acme-journal.org/index.php/acme/article/view/1735.

Reynolds K., Cohen N., 2016. Beyond the kale: urban agriculture and social justice activism in New York city, Athens, The University of Georgia Press.

Rosol M., 2012. Community volunteering as neoliberal strategy? Green space production in Berlin, Antipode, 44, 1, 239-257, https://doi.org/10.1111/j.1467-8330.2011.00861.x.

Routledge P., 1996. The third space as critical engagement, Antipode, 28, 4, 399-419, https://doi.org/10.1111/ j.1467-8330.1996.tb00533.x.

Séchet R., Veschambre V., 2006. Introduction générale, in Séchet R., Veschambre V.(Ed.), Penser et faire la géographie sociale: contributions à une épistémologie de la géographie sociale, Rennes, Presses universitaires de Rennes, 7-24.

Soulard C.-T., Rixen A., Scheromm P., 2018. Practicing action-research in the French context: a critical reflection of working relationships between researchers and local actors in a collaborative project on agroecology. Communication à la session Action Research in International Critical Urban Agrifood Systems Geographies, $A A G$ 18, 10 avril, La Nouvelle Orléans.

Soulié C. (Ed.), 2012. Un mythe à détruire? Origines et destin $d u$ Centre universitaire expérimental de Vincennes, SaintDenis, Presses universitaires de Vincennes.

Slocum R., Cadieux K.V., Blumberg R., 2016. Solidarité, espace et «race»: vers des géographies de la justice alimentaire, Justice spatiale/Spatial Justice, 9, www.jssj. org/article/solidarite-espace-et-race-vers-des-geographiesde-la-justice-alimentaire/.

Tax S., 1975. Action anthropology, Current Anthropology, 16, 4, 514-517, https://doi.org/10.1086/201616.

Tornaghi C., 2014. Critical geography of urban agriculture, Progress in Human Geography, 38, 4, 551-567, https://doi. org/10.1177/0309132513512542.

Tornaghi C., Certomà C. (Eds), 2019. Urban gardening as politics, Londres/New York, Routledge.

Torre M.E., Fine M., 2011. A wrinkle in time: tracing a legacy of public science through community self-surveys and participatory action research, Journal of Social Issues, 67, 1, 106-121, https://doi.org/10.1111/j.1540-4560.2010.01686.x.

Tuck E., 2009. Suspending damage: a letter to communities, Harvard Educational Review, 79, 3, 409-428, https://doi. org/10.17763/haer.79.3.n0016675661t3n15.

Vidal R., Fleury A., 2009. Aménager les relations entre la ville et l'agriculture. De nouveaux enjeux territoriaux et une nouvelle approche "agriurbaniste », Urbia, 8, 127-142, www.unil.ch/files/live/sites/ouvdd/files/shared/URBIA/ urbia_08/urbia_08_p127_142.pdf.

Citation de l'article: Salomon Cavin J., Boisvert V., Ranocchiari S., Dusserre-Bresson Q., Poulot M. L'engagement militant dans la recherche en agriculture urbaine. Réflexions sur le contexte français au miroir du scholar activism nord-américain. Nat. Sci. Soc. 29, 3, 288-298. 